$12-1-1997$

\title{
Three-Dimensional Camera Calibration Technique for Stereo Imaging Velocimetry Experiments
}

\author{
Mark D. Bethea \\ James A. Lock \\ Cleveland State University, j.lock@csuohio.edu \\ Frank Merat
}

Paul Crouser

Follow this and additional works at: https://engagedscholarship.csuohio.edu/sciphysics_facpub

Part of the Physics Commons

How does access to this work benefit you? Let us know!

Publisher's Statement

Copyright 1997 Society of Photo-Optical Instrumentation Engineers. One print or electronic copy may be made for personal use only. Systematic reproduction and distribution, duplication of any material in this paper for a fee or for commercial purposes, or modification of the content of the paper are prohibited. Available from publisher at http://dx.doi.org/10.1117/1.601584.

\section{Original Citation}

Bethea, Mark D., James A. Lock, Frank Merat and Paul Crouser. "Three-Dimensional Camera Calibration Technique for Stereo Imaging Velocimetry Experiments." Optical Engineering 36 (1997): 3445-3454.

\section{Repository Citation}

Bethea, Mark D.; Lock, James A.; Merat, Frank; and Crouser, Paul, "Three-Dimensional Camera Calibration Technique for Stereo Imaging Velocimetry Experiments" (1997). Physics Faculty Publications. 27.

https://engagedscholarship.csuohio.edu/sciphysics_facpub/27

This Article is brought to you for free and open access by the Physics Department at EngagedScholarship@CSU. It has been accepted for inclusion in Physics Faculty Publications by an authorized administrator of EngagedScholarship@CSU. For more information, please contact library.es@csuohio.edu. 


\section{Three-dimensional camera calibration technique for stereo imaging velocimetry experiments}

\author{
Mark D. Bethea \\ NASA Lewis Research Center \\ Processing Science and Technology Branch \\ Cleveland, Ohio 44135 \\ E-mail: msbeth@sarah.lerc.nasa.gov \\ James A. Lock \\ Cleveland State University \\ Department of Physics \\ Cleveland, Ohio 44115 \\ Frank Merat, MEMBER SPIE \\ Case Western Reserve University \\ Department of Electrical Engineering and \\ Applied Physics \\ Cleveland, Ohio 44106 \\ Paul Crouser \\ Case Western Reserve University \\ Department of Computer Engineering and \\ Science \\ Cleveland, Ohio 44106
}

\begin{abstract}
A three-dimensional camera calibration technique is developed by combining two, 2-D camera calibrations for an orthogonal stereo viewing geometry. The left camera view ( $Y Z$ view) and the right camera view ( $X Z$ view) are calibrated separately and then combined to produce an $X Y Z$ (3-D) calibration routine. Our technique employs three parallel calibration planes. One is placed along the main diagonal of the cubic experimental chamber, and the other two planes are placed known distances in front of it and behind it within the chamber. Both cameras view the calibration points on the planes simultaneously. Given the coordinates of a number of points, we use a physical model to determine the exact pixel locations of the calibration points. After inverting the model equations, we input the absolute coordinates and measured pixel locations into a least-squares fitting algorithm to obtain the experimental camera parameters for each camera individually. We then combine the two camera views via a ray-tracing method. We calibrated 3 -in. ${ }^{3}$ $\left(7.62-\mathrm{mm}^{3}\right), \quad 4-$ in. $^{3}\left(10.16-\mathrm{mm}^{3}\right), \quad 5-\mathrm{in}^{3}\left(12.70-\mathrm{mm}^{3}\right)$, and $6-\mathrm{in.}^{3}$ $\left(15.24-\mathrm{mm}^{3}\right)$ chambers with accuracies between 1.66 and 2.01 pixels ( 0.60 and $0.77 \%$ of full field), 1.26 and 1.86 pixels $(0.43$ and $0.63 \%$ of full field), 1.16 and 1.34 pixels (0.33 and $0.39 \%$ of full field), and 1.91 and 2.49 pixels $(0.59$ and $0.77 \%$ of full field), respectively, using our 3-D camera calibration routine. $\odot 1997$ Society of Photo-Optical Instrumentation Engineers. [S0091-3286(97)03012-2]

: $x$

Subject terms: stereo imaging velocimetry; three-dimensional camera calibration technique; stereo imaging geometry; computer vision; machine vision; particle imaging velocimetry.
\end{abstract}

Paper 25057 received May 23, 1997; accepted for publication Aug. 11, 1997.

\section{Introduction}

Three-dimensional camera calibration for stereo imaging velocimetry is a process by which one determines the geometrical and experimental parameters of a flow chamber for the purpose of carrying out flow velocity measurements. The geometrical parameters are the internal camera characteristics such as focal length and pixel size. The experimental parameters are the positions of seed particles entrained in the flow and the orientation of the cameras relative to a certain world coordinate system. Camera calibration is the most important aspect of any computer vision experiment since it serves as a lower limit for determining system accuracy. It is important that this routine be as accurate as possible, since in any experiment, we can never obtain a smaller error in particle position than that dictated by our camera calibration procedures.

There have been a number of different approaches to 3-D camera calibration. Tsai ${ }^{1,2}$ reported a versatile camera calibration technique using off-the-shelf cameras. He also discussed the existing camera calibration literature and the advantages and disadvantages of each existing method. Weng et al. ${ }^{3}$ reported a more up to date comparison of existing methods and implemented a nonlinear iterative scheme that is as accurate as any other previously published 3-D camera calibration procedure. They also introduced a measure of intrinsic calibration error, the normal- ized stereo calibration error (NSCE), which represents the ratio of the mean lateral triangulation error to the lateral pixel quantization error. In their application, the NSCE represented the combined effects of the two camera calibrations. Martins et al. ${ }^{4}$ incorporated the use of two calibration planes, one at the front and one at the back of the experimental chamber, to implement their camera calibration routine. Kamgar-Parsi and Eastman ${ }^{5}$ discussed the practical difficulties in the calibration of a two-camera stereo system in an uncontrolled environment. Adamczyk and Rimai ${ }^{6}$ developed a camera calibration routine to be used in the reconstruction of 3-D flows from two orthogonal views in a cylindrical volume. They use transformation functions that related test-section locations to their corresponding video coordinates, correcting for optical distortions and properly scaling the results. Racca and Dewey ${ }^{7}$ presented a calibration method for automatic particle tracking in a 3-D flow field using a series of mirrors to convert two orthogonal views to a side-by-side format. Koybayshi et al. ${ }^{8}$ adapted stereo photogrammetry for multipoint $3-\mathrm{D}$ velocity measurements of a fluid between two parallel counter-rotating cylinders. They discussed a calibration technique for nonmetric cameras using an absolute coordinate system, camera coordinate system, and a photographic plane. Nishino et al. ${ }^{9}$ implemented a 3-D particle tracking technique in a volume using three cameras instead of two. Using their three-camera system, the reconstruction of 3-D particle po- 


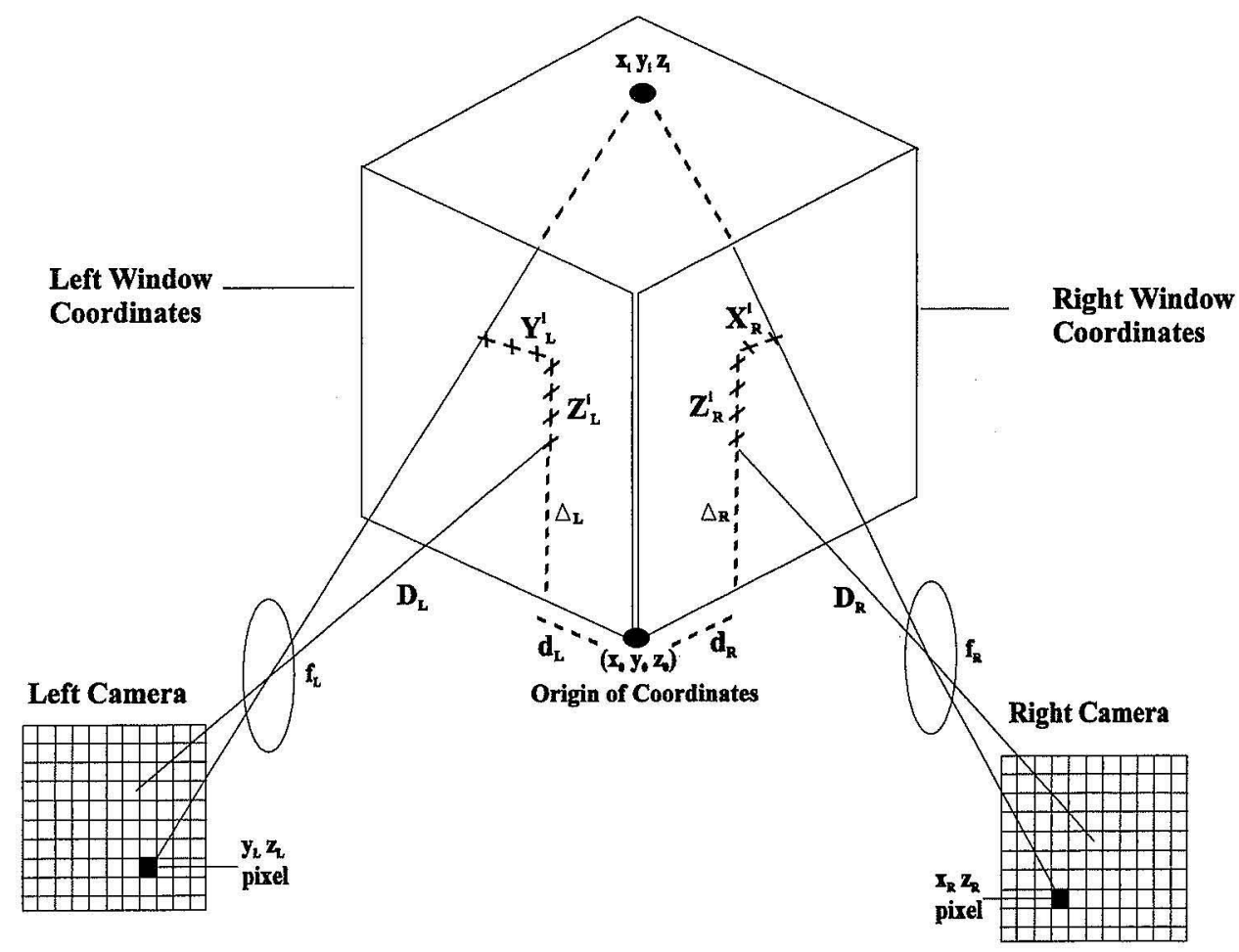

Fig. 1 Camera calibration nomenclature. Right camera perspective (the left camera perspective is analogous): $x_{i}, y_{i}, z_{i}=$ absolute $x, y, z$ coordinates of particle $i . X_{R}^{i} Z_{R}^{i}=$ window coordinates of particle $i$ on the right face of the chamber. $x_{R}^{i} z_{R}^{i}=$ pixel coordinates of particle $i$ as seen by the right camera. $f_{R}=$ effective focal length of the right camera. $D_{R}=$ effective distance between the right camera and the face of the chamber. $d_{R}=$ horizontal distance of the right camera axis from the origin. $\Delta_{R}=$ vertical distance of the right camera axis from the origin. $C_{R}=$ camera dependent constant with the units millimeters per pixel.

$$
\text { tha }
$$

sitions and camera parameters were determined and the relationship of the absolute and photographic coordinate system was given. Miller et al. ${ }^{10}$ briefly discussed 3-D camera calibration as it relates to stereo imaging velocimetry experiments. Their technique used a least-squares data fitting routine to achieve calibration results based on using three calibration planes in the measurement volume. This paper describes in detail the camera calibration technique mentioned in Refs. 10, 11, and 12 .

Our approach builds on the successes of this research to construct an efficient and accurate 3-D camera calibration routine. We develop a theoretical formulation, physical model, and experimental model (cubical chamber with flat sides) and compare the results to published work. Our goal is to provide the capability for accurately calibrating a volume with two orthogonal cameras. Our calibration technique uses a polynomial approximation instead of a nonlinear iterative scheme or an artificial neural network approximation. We find this polynomial approximation 3-D calibration technique to be of comparable accuracy to other published work on 3-D camera calibration, and to be much faster than either nonlinear or artificial neural network techniques for implementation in stereo imaging applications. This method has been tested and is currently being used for laboratory and industrial fluid flow analysis.

\section{Stereo Imaging Geometry}

Our camera calibration geometry is shown in Fig. 1. The origin of the absolute coordinate system is placed such that it is in the lower left-hand comer of the experimental flow chamber, when viewed by the right camera, and in the lower right-hand corner of the chamber, when viewed by the left camera. This geometry defines the right and left camera views. Here $\left(x_{i}, y_{i}, z_{i}\right)$ are the coordinates of particle $i$ with respect to the origin. A ray of light leaves particle $i$ and strikes the right camera CCD array at the pixel location $\left(x_{R}^{i}, z_{R}^{i}\right)$ with respect to the center of the array, which is taken to be on the symmetry axis of the camera lens. Another ray of light leaves particle $i$ and strikes the left camera $\mathrm{CCD}$ array at the pixel location $\left(y_{L}^{i}, z_{L}^{i}\right)$ with respect to the center of the array which is taken to be on the symmetry axis of the camera lens. Distances $D_{R}$ and $D_{L}$ are the effective distances of the cameras from the left and right faces of the particle chamber, and $f_{R}$ and $f_{L}$ are the effective focal lengths of the cameras. The left camera axis is a distance $\Delta_{L}-\Delta_{R}$ higher than the right camera axis and intersects the right face of the particle chamber a distance $d_{L}$ from the edge with respect to the left face. The ray going from particle $i$ to the right camera crosses the right face of the particle chamber a distance $Z_{R}^{i}$ above and $X_{R}^{i}$ to the right of the axis of the right camera. The ray going from particle $i$ to the left camera crosses the left face of the particle chamber a distance $Z_{L}^{i}$ above and $Y_{L}^{i}$ to the left of the axis of the left camera. During calibration, all we know are the absolute coordinates $\left(x_{i}, y_{i}, z_{i}\right)$ and pixel readouts 
$\left(x_{R}^{i}, z_{R}^{i}\right)$ and $\left(y_{L}^{i}, z_{L}^{i}\right)$ of our predetermined calibration points. Our goal is to determine $f_{L}, D_{L}, \Delta_{L}, d_{L}, f_{R}, D_{R}$, $\Delta_{R}$, and $d_{R}$ by least-squares data fitting so that during camera operation after the calibration procedure is completed, we can determine the absolute coordinates $\left(x_{j}, y_{j}, z_{j}\right)$ of a seed particle entrained in the flow given only its pixel positions $\left(x_{R}^{j}, z_{R}^{j}\right)$ and $\left(y_{L}^{j}, z_{L}^{j}\right)$ on the camera focal planes.

\section{Least-Squares Data Fitting}

Let the inputs $x_{k}$ for $1 \leqslant k \leqslant M$ and the output $y$ of a physical situation be described by the linear equation

$y=\sum_{k=1}^{M} \alpha_{k} x_{k}$

where $y$ is the dependent variable, $\alpha_{k}$ are constants of proportionality, and $x_{k}$ are independent variables.

Assume that in our experiment we measure $\left(x_{1}, x_{2}, \ldots, x_{M}, y\right)$ for $Q$ different situations and we wish to determine the values of the constants of proportionality. Let $x_{k}^{i}$ represent the observed value of $x_{k}$ in the $i$ 'th measurement, and let $y^{i}$ represent the observed value of $y$ in the $i$ 'th measurement. Also, let

$y_{\text {expected }}^{i}=\sum_{k=1}^{M} \alpha_{k} x_{k}^{i}$

be the expected value of $y$ based on the observed values of $\left(x_{1}^{i}, x_{2}^{i}, \ldots, x_{M}^{i}\right)$ in the $i$ 'th measurement. Then,

$\Delta^{i}=y^{i}-y_{\text {expected }}^{i}$

is the difference between the observed and expected value of $y$ in the $i$ 'th measurement, and

$\Delta=\sum_{i=1}^{Q}\left(\Delta^{i}\right)^{2}=\sum_{i=1}^{Q}\left(y^{i}-\sum_{k=1}^{M} \alpha_{k} x_{k}^{i}\right)\left(y^{i}-\sum_{l=1}^{M} \alpha_{l} x_{l}^{i}\right)$

is the total accumulated difference squared over all the $Q$ measurements. In the method of least-squares data fitting, we make the total accumulated difference squared a minimum, i.e.,

$\frac{\partial \Delta}{\partial \alpha_{j}}=0$ for $i \leqslant j \leqslant M$.

Performing the derivatives, we then obtain

$\left(\sum_{i=1}^{Q} x_{j}^{i} y^{i}\right)=\sum_{k=1}^{M} \alpha_{k}\left(\sum_{i=1}^{Q} x_{k}^{i} x_{j}^{i}\right) \quad$ for $1 \leqslant j \leqslant M$.

This is a system of $M$ linear equations in the $M$ unknowns for $\alpha_{1}, \alpha_{2}, \ldots, \alpha_{M}$, which can be solved by matrix inversion. In Secs. 6 and 7, we use this least-squares procedure to solve for the camera calibration coefficients.

\section{Physical Model}

The coordinates of a particle in space with respect to some fixed laboratory coordinate system are $x, y$, and $z ; X_{R}$ and $Z_{R}$ are the right camera coordinates of the particle on the face of the chamber, as shown in Fig. 1; and $x_{R}$ and $z_{R}$ are the pixel coordinates of the particle from the right camera perspective. The left camera perspective is analogous. Consider the following oversimplified model of the experimental chamber-camera system.

1. We assume that the experimental chamber is filled with air and that the refraction that occurs at the flat windows of the chamber is minimal. This is a good approximation if the windows are thin. The relation between the absolute coordinates and the window coordinates of Fig. 1 is then

$$
\begin{aligned}
& x=d_{R}+X_{R}+\frac{X_{R} y}{D_{R}}, \\
& z=\Delta_{R}+Z_{R}+\frac{Z_{R} y}{D_{R}} .
\end{aligned}
$$

This portion of our model deals only with the chamber and relates $x, y$, and $z$ to $X_{R}$ and $Z_{R}$.

2. The camera reference directions may be misaligned with respect to the axes of the laboratory coordinate system attached to the experimental chamber. We assume the symmetry axis of the camera lens is perpen-

" " dicular to the nearest chamber face, but that the camera pixel axes are rotated by the angle $\phi$ with respect to the lab axes. Then

$x_{R}^{\text {ideal }}=x_{R}^{\text {actual }} \cos \phi-z_{R}^{\text {actual }} \sin \phi$,
$z_{R}^{\text {ideal }}=x_{R}^{\text {actual }} \sin \phi+z_{R}^{\text {actual }} \cos \phi$,

where $x_{R}^{\text {ideal }}$ and $z_{R}^{\text {ideal }}$ are parallel to the lab axes.

3. If the camera has a radially symmetric magnification distortion, the pixel coordinates and the right window coordinates of Fig. 1 are related by

$$
\begin{aligned}
x_{R}^{\text {ideal }}= & \frac{f_{R}}{C_{R} D_{R}} X_{R}\left[1+\lambda\left(X_{R}^{2}+Z_{R}^{2}\right)^{1 / 2}+\lambda^{\prime}\left(X_{R}^{2}+Z_{R}^{2}\right)\right. \\
& \left.+\lambda^{\prime \prime}\left(X_{R}^{2}+Z_{R}^{2}\right)^{3 / 2}+\cdots\right], \\
z_{R}^{\text {ideal }}= & \frac{f_{R}}{C_{R} D_{R}} Z_{R}\left[1+\lambda\left(X_{R}^{2}+Z_{R}^{2}\right)^{1 / 2}+\lambda^{\prime}\left(X_{R}^{2}+Z_{R}^{2}\right)\right. \\
& \left.+\lambda^{\prime \prime}\left(X_{R}^{2}+Z_{R}^{2}\right)^{3 / 2}+\ldots\right],
\end{aligned}
$$

where $f_{R} /\left(C_{R} D_{R}\right)$ is the nominal camera magnification, $C_{R}=$ pixel size for the right camera in millimeters per pixel width and where $\lambda, \lambda^{\prime}, \lambda^{\prime \prime}$, etc. are related to the various Seidell aberration coefficients. For simplicity, we retain the term of order $\lambda$ but neglect the $\lambda^{\prime}$ and $\lambda^{\prime \prime}$ terms.

This portion of our model only deals with the camera and relates $X_{R}$ and $Z_{R}$ to $x_{R}$ and $z_{R}$. We now combine ideas 1 to 3 to obtain the pixel coordinates as functions of the absolute coordinates for our physical model. By combining Eqs. (8) and (9) we obtain 


$$
\begin{aligned}
x_{R}^{\text {actual }}= & \frac{f_{R}}{C_{R} D_{R}}\left[1+\lambda\left(X_{R}^{2}+Z_{R}^{2}\right)^{1 / 2}\right]\left(X_{R} \cos \phi+Z_{R} \sin \phi\right), \\
z_{R}^{\text {actual }}= & \frac{f_{R}}{C_{R} D_{R}}\left[1+\lambda\left(X_{R}^{2}+Z_{R}^{2}\right)^{1 / 2}\right]\left(-X_{R} \sin \phi\right. \\
& \left.+Z_{R} \cos \phi\right) .
\end{aligned}
$$

But, the absolute coordinates and the window coordinates of the particle are related via Eq. (7) by

$X_{R}=\frac{x-d_{R}}{1+y / D_{R}}$

$Z_{R}=\frac{z-\Delta_{R}}{1+y / D_{R}}$

Therefore, given the absolute coordinates of the particle in space, the pixel coordinates of the particle's image in our simplified model are

$$
\begin{aligned}
x_{R}^{\text {actual }}= & \frac{f_{R}}{C_{R} D_{R}}\left\{1+\frac{\lambda}{1+y / D_{R}}\left[\left(x-d_{R}\right)^{2}\right.\right. \\
& \left.\left.+\left(z-\Delta_{R}\right)^{2}\right]^{1 / 2}\right\} \frac{1}{1+y / D_{R}}\left[\left(x-d_{R}\right) \cos \phi\right. \\
& \left.+\left(z-\Delta_{R}\right) \sin \phi\right], \\
z_{R}^{\text {actual }}= & \frac{f_{R}}{C_{R} D_{R}}\left\{1+\frac{\lambda}{1+y / D_{R}}\left[\left(x-d_{R}\right)^{2}\right.\right. \\
& \left.\left.+\left(z-\Delta_{R}\right)^{2}\right]^{1 / 2}\right\} \frac{1}{1+y / D_{R}}\left[-\left(x-d_{R}\right) \sin \phi\right. \\
& \left.+\left(z-\Delta_{R}\right) \cos \phi\right] .
\end{aligned}
$$

Hereafter, the superscripts "actual" are omitted from the pixel coordinates. Our model for the camera is oversimplified, but as it turns out, is already complicated enough so that we cannot invert Eq. (12) analytically to obtain $x$ and $z$ in terms of $x_{R}, z_{R}$, and $y$. In spite of our inability to carry out the inversion exactly, performing some type of an inversion of Eq. (12) is the single most important element in 2-D camera calibration.

\section{Approximate Inversion Procedure for the Theoretical Model}

In this subsection, we invert Eq. (12) in an approximate way. Let

$F=1+\lambda\left(X_{R}^{2}+Z_{R}^{2}\right)^{1 / 2}$.

Then, Eq. (10) can be wrriten as

$\frac{x_{R}}{F} \frac{C_{R} D_{R}}{f_{R}}=X_{R} \cos \phi+Z_{R} \sin \phi$,

$\frac{z_{R}}{F} \frac{C_{R} D_{R}}{f_{R}}=-X_{R} \sin \phi+Z_{R} \cos \phi$.
Equations (14) can then be solved for $X_{R}$ and $Z_{R}$ [as in Eq. (10)] to give

$X_{R}=\frac{C_{R} D_{R}}{f_{R} F}\left(x_{R} \cos \phi-z_{R} \sin \phi\right)$,

$Z_{R}=\frac{C_{R} D_{R}}{f_{R} F}\left(x_{R} \sin \phi+z_{R} \cos \phi\right)$.

Equations (15) are not a true inverse of Eqs. (14) since $F$ contains $X_{R}$ and $Z_{R}$. For small $\lambda$, however, some simplification does occur since we may expand $1 / F$ in a Taylor series in powers of $\lambda$. Explicitly, we have

$$
\begin{aligned}
\frac{1}{F} & =\frac{1}{1+\lambda\left(X_{R}^{2}+Z_{R}^{2}\right)^{1 / 2}} \\
& \approx 1-\lambda\left(X_{R}^{2}+Z_{R}^{2}\right)^{1 / 2}+\lambda^{2}\left(X_{R}^{2}+Z_{R}^{2}\right)-\cdots .
\end{aligned}
$$

Substituting $X_{R}$ and $Z_{R}$ from Eqs. (15) into Eq. (16) and again expanding the new $1 / F$ terms, we obtain

$$
\begin{aligned}
\frac{1}{F} \approx & 1-\lambda\left(\frac{C_{R} D_{R}}{f_{R}}\right)\left(x_{R}^{2}+z_{R}^{2}\right)^{1 / 2}+2 \lambda^{2}\left(\frac{C_{R} D_{R}}{f_{R}}\right)^{2} \\
& \times\left(x_{R}^{2}+z_{R}^{2}\right)+\cdots .
\end{aligned}
$$

Thus, substituting Eq. (17) back into Eqs. (15), the window coordinates $X_{R}$ and $Z_{R}$ of Fig. 1 can be written in terms of the pixel coordinates as the infinite series

$$
\begin{aligned}
X_{R}= & \frac{C_{R} D_{R}}{f_{R}}\left(x_{R} \cos \phi-z_{R} \sin \phi\right)\left[1-\lambda\left(\frac{C_{R} D_{R}}{f_{R}}\right)\right. \\
& \left.\times\left(x_{R}^{2}+z_{R}^{2}\right)^{1 / 2}+2 \lambda^{2}\left(\frac{C_{R} D_{R}}{f_{R}}\right)^{2}\left(x_{R}^{2}+z_{R}^{2}\right) \cdots\right]
\end{aligned}
$$

$$
\begin{aligned}
Z_{R}= & \frac{C_{R} D_{R}}{f_{R}}\left(x_{R} \sin \phi+z_{R} \cos \phi\right)\left[1-\lambda\left(\frac{C_{R} D_{R}}{f_{R}}\right)\right. \\
& \left.\times\left(x_{R}^{2}+z_{R}^{2}\right)^{1 / 2}+2 \lambda^{2}\left(\frac{C_{R} D_{R}}{f_{R}}\right)^{2}\left(x_{R}^{2}+z_{R}^{2}\right) \cdots\right] .
\end{aligned}
$$

For small $\lambda$, the series is rapidly convergent and Eqs. (18) can be considered as the inverse of Eqs. (14). Using Eqs. (7), the particle absolute coordinates may be written in terms of the pixel coordinates for our model as 


$$
\begin{aligned}
x= & d_{R}+\left(1+\frac{y}{D_{R}}\right) X_{R} \\
= & d_{R}+\left(\frac{C_{R} D_{R}}{f_{R}}\right)\left(x_{R} \cos \phi-z_{R} \sin \phi\right. \\
& \left.+\frac{x_{R}}{D_{R}} y \cos \phi-\frac{z_{R}}{D_{R}} y \sin \phi\right)\left[1-\lambda\left(\frac{C_{R} D_{R}}{f_{R}}\right)\right. \\
& \left.\times\left(x_{R}^{2}+z_{R}^{2}\right)^{1 / 2}+2 \lambda^{2}\left(\frac{C_{R} D_{R}}{f_{R}}\right)^{2}\left(x_{R}^{2}+z_{R}^{2}\right) \cdots\right], \\
z= & \left.\Delta_{R}+\left(1+\frac{y}{D_{R}}\right) Z_{R}\right] \\
= & \Delta_{R}+\left(\frac{C_{R} D_{R}}{f_{R}}\right)\left(x_{R} \sin \phi+z_{R} \cos \phi+\frac{x_{R}}{D_{R}} y \sin \phi\right. \\
& \left.+\frac{z_{R}}{D_{R}} y \cos \phi\right)\left[1-\lambda\left(\frac{C_{R} D_{R}}{f_{R}}\right)\left(x_{R}^{2}+z_{R}^{2}\right)^{1 / 2}\right. \\
& +2 \lambda^{2}\left(\frac{C_{R} D_{R}}{f_{R}}\right)^{2}\left(x_{R}^{2}+z_{R}^{2}\right) \cdots \\
&
\end{aligned}
$$

As mentioned previously, our physical model of the light propagation from the test particle at $\left(x_{i}, y_{i}, z_{i}\right)$ to the pixel coordinates $\left(x_{R}^{i}, z_{R}^{i}\right)$ and $\left(y_{L}^{i}, z_{L}^{i}\right)$ is greatly oversimplified. The test particles are, in actuality, entrained in a flowing liquid in the scattering chamber. As a result, light rays leaving the chamber are refracted at its walls. Further, the cameras may be misaligned such that the symmetry axes of the lenses are not exactly perpendicular to the faces of the chamber. The camera lenses may have additional aberrations besides the radial distortion modeled in Eqs. (9). We wish to include these additional realistic possibilities in our camera calibration model of Eqs. (19). We do this in the following way. First, we approximate the square root in Eqs. (19) by

$\left(x_{R}^{2}+z_{R}^{2}\right)^{1 / 2} \approx\left|x_{R}\right|+\left|z_{R}\right|+\gamma\left(\left|x_{R}\right|\left|z_{R}\right|\right)^{1 / 2}$,

where

$\gamma=\sqrt{2}-2$

This expression is exact on the $x_{R}$ axis, the $z_{R}$ axis, and on the lines $x_{R}= \pm z_{R}$. Since Eq. (20) is multiplied by the small number $\lambda$ in Eqs. (19), the replacement of the square root by the magnitude factors in Eq. (20) is expected to introduce only a small amount of error into the calibration procedure. Substituting Eq. (20) into Eqs. (19) we then obtain

$$
\begin{aligned}
x \approx & A_{0}+A_{1} x_{R}-A_{2} z_{R}+A_{3} x_{R} y-A_{4} z_{R} y-A_{5} x_{R}\left|x_{R}\right| \\
& -A_{6} x_{R}\left|z_{R}\right|+A_{7}\left|x_{R}\right| z_{R}+A_{8}\left|z_{R}\right| z_{R}-A_{9} x_{R}\left(\left|x_{R}\right|\left|z_{R}\right|\right)^{1 / 2} \\
& +A_{10^{2}}\left(\left|x_{R}\right|\left|z_{R}\right|\right)^{1 / 2}-A_{11} x_{R}\left|x_{R}\right| y-A_{12} x_{R}\left|z_{R}\right| y \\
& +A_{13} z_{R}\left|x_{R}\right| y+A_{14} z_{R}\left|z_{R}\right| y-A_{15} x_{R}\left(\left|x_{R}\right|\left|z_{R}\right|\right)^{1 / 2} y \\
& +A_{16} z_{R}\left(\left|x_{R}\right|\left|z_{R}\right|\right)^{1 / 2} y+A_{17} x_{R}^{3}-A_{18} x_{R}^{2} z_{R}+A_{19} x_{R} z_{R}^{2} \\
& -A_{20} z_{R}^{3}+A_{21} x_{R}^{3} y-A_{22} x_{R}^{2} z_{R} y+A_{23} x_{R} z_{R}^{2} y-A_{24} z_{R}^{3} y \\
& +\cdots, \\
z \approx & B_{0}+B_{1} x_{R}+B_{2} z_{R}+B_{3} x_{R} y+B_{4} z_{R} y-B_{5} x_{R}\left|x_{R}\right| \\
& -B_{6} x_{R}\left|z_{R}\right|-B_{7}\left|x_{R}\right| z_{R}-B_{8}\left|z_{R}\right| z_{R}-B_{9} x_{R}\left(\left|x_{R}\right|\left|z_{R}\right|\right)^{1 / 2} \\
& -B_{10} z_{R}\left(\left|x_{R}\right|\left|z_{R}\right|\right)^{1 / 2}-B_{11} x_{R}\left|x_{R}\right| y-B_{12} x_{R}\left|z_{R}\right| y \\
& -B_{13} z_{R}\left|x_{R}\right| y-B_{14} z_{R}\left|z_{R}\right| y-B_{15} x_{R}\left(\left|x_{R}\right|\left|z_{R}\right|\right)^{1 / 2} y \\
& -B_{16} z_{R}\left(\left|x_{R}\right|\left|z_{R}\right|\right)^{1 / 2} y+B_{17} x_{R}^{3}+B_{18} x_{R}^{2} z_{R}+B_{19} x_{R} z_{R}^{2} \\
& +B_{20} z_{R}^{3}+B_{21} x_{R}^{3} y+B_{22} x_{R}^{2} z_{R} y+B_{23} x_{R} z_{R}^{2} y+B_{24} z_{R}^{3} y \\
& +\cdots
\end{aligned}
$$

where

$A_{0}=d_{R}$,

$A_{1}=\frac{{ }_{4} C_{R} C_{R}}{f_{R}} \cos \phi$,

$A_{2}=\frac{C_{R} D_{R}}{f_{R}} \sin \phi$,

and so forth. If refraction at the surface of the scattering chamber occurs, if the camera axes are misaligned with respect to the faces of chamber, or if the camera lenses possess additional aberrations, we claim that Eqs. (22) still accurately describe the scattering chamber-camera geometry. But the geometrical meaning of the coefficients $A_{0} \ldots A_{24}$ and $B_{0} \ldots B_{24}$ is no longer given by Eqs. (23). The necessity of approximating $\left(x_{R}^{2}+z_{R}^{2}\right)^{1 / 2}$ by Eq. (20) now becomes evident. The $\left(x_{R}^{2}+z_{R}^{2}\right)^{1 / 2}$ factor describes a radially symmetric situation such as radial distortion or spherical aberration. But if the lens axis of a camera is misaligned so that it is not perpendicular to a face of the scattering chamber, one side of the face is closer to the camera resulting in a larger magnification while the other side of the face is farther from the camera resulting in a smaller magnification. The breaking of the radial symmetry by this left-right variable magnification is accomplished in our model by replacing $\left(x_{R}^{2}+z_{R}^{2}\right)^{1 / 2}$ by Eq. (20) and letting the coefficients of $\left|x_{R}\right|,\left|z_{R}\right|$, and $\left(\left|x_{R}\right|\left|z_{R}\right|\right)^{1 / 2}$ take on undetermined values, as in Eqs. (22). Equations (22) are 25-term polynomial approximations for $x$ and $z$ (right camera) in terms of $x_{R}, z_{R}$ and $y$. Using the same technique, the left camera calibration equation for $y$ and $z$, yields a 25-term polynomial approximation for $y$ and $z$ (left camera) in terms of $y_{L}, z_{L}$, and $x$. We consider five different truncations of Eqs. (22) and assess the intrinsic theoretical error in each one. Model 
1 uses the first 9 terms of the polynomials in Eqs. (22), model 2 uses the first 11 terms, model 3 uses the first 17 terms, model 4 uses the first 21 terms, and model 5 uses all 25 terms of the polynomials in Eqs. (22). The purpose of this test is to determine how many terms in the series are required for convergence for realistic stereo imaging velocimetry (SIV) situations.

\section{Theoretical Analysis}

In this section, we mathematically test the accuracy of our approximate inversion method in the following way. Given values of $x, y$, and $z$, we calculate the pixel coordinates $\left(x_{R}, z_{R}\right)$ with the exact equations [Eqs. (12)]. We then input these values of $x_{R}$ and $z_{R}$ into Eqs. (22) to obtain first the values of $A_{0} \ldots B_{24}$, and then the approximate values of $x$ and $z$. We then compare these approximate values with the original values. This gives us the intrinsic accuracy of our approximate inversion procedure for the right camera. The left camera accuracy (using $y_{L}$ and $z_{L}$ to obtain the approximate values of $y$ and $z$ ) is determined in the same manner.

We chose the following camera parameters to test our theoretical polynomial approximation. The values chosen are arbitrary, but are representative for our experimental model. We chose a 5 -in. ${ }^{3}\left(127-\mathrm{mm}^{3}\right)$ chamber, a pixel size of $12 \mu \mathrm{m}$, a pixel array size of $6.144 \mathrm{~mm}^{2}$ on a $512 \times 512$ array, and we place the camera $12 \mathrm{in.}(304.8 \mathrm{~mm}$ ) away from the front edge of the chamber. We image the entire chamber onto the CCD array. This gives a focal length of $f_{R}=14.75 \mathrm{~mm}$, and the half angle of the field of view is $11.77 \mathrm{deg}$. Assume that the camera axis is perfectly centered on the face of the chamber so that $d_{R}=63.5 \mathrm{~mm}$. (half the width of the chamber) and $\Delta_{R}=63.5 \mathrm{~mm}$ (half the height of the chamber). Let the size of the radial distortion be $5 \%$ of the field of view at the corners of the array. The distortion at the corner of the $\mathrm{CCD}$ array is then $(256$ pixels $\sqrt{2})(.05)=18.1$ pixels and

$\lambda=5.57 \times 10^{-4} \mathrm{~mm}^{-1}$.

Let $\phi=3 \mathrm{deg}$ be the rotation of the CCD axes with respect to the chamber axes. Then

$\frac{f_{R}}{C_{R} D_{R}}=\frac{14.7456}{(0.012)(304.8)}=14.03150 \mathrm{~mm}^{-1}$.

We chose 25 reference points in 5 rows of 5 placed along the main diagonal of the experimental chamber. Thus, the vertical position of the points was $[10.0+26.75(i$ $-1)] \mathrm{mm}$ for $1 \leqslant i \leqslant 5$ and the horizontal position of the points was $[10.0+37.5(j-1)] \mathrm{mm}$ for $1 \leqslant j \leqslant 5$. The absolute coordinates of the reference points were then

$x_{i j}=0.71[10.0+37.5(j-1)] \mathrm{mm}$,

$y_{i j}=0.71[10.0+37.5(j-1)] \mathrm{mm}$,

$z_{i j}=[10.0+26.75(i-1)] \mathrm{mm}$,

for $1 \leqslant i \leqslant 5$ and $1 \leqslant j \leqslant 5$.
Table 1 Absolute and pixel coordinates for theoretical analysis.

\begin{tabular}{|c|c|c|c|c|c|}
\hline$i$ & $x_{i}(\mathrm{~mm})$ & $y_{i}(\mathrm{~mm})$ & $z_{i}(\mathrm{~mm})$ & $x_{R}^{\prime}$ (pixel no.) & $z_{R}^{i}$ (pixel no.) \\
\hline 0 & 7.10 & 7.10 & 10.00 & -243 & -207 \\
\hline 1 & 33.72 & 33.72 & 10.00 & -122 & -194 \\
\hline 2 & 60.35 & 60.35 & 10.00 & -21 & -184 \\
\hline 3 & 86.97 & 86.97 & 10.00 & 66 & -176 \\
\hline 4 & 113.60 & 113.60 & 10.00 & 143 & -170 \\
\hline 5 & 7.10 & 7.10 & 36.75 & -235 & -97 \\
\hline 6 & 33.72 & 33.72 & 36.75 & -115 & -93 \\
\hline 7 & 60.35 & 60.35 & 36.75 & -15 & -90 \\
\hline 8 & 86.97 & 86.97 & 36.75 & 70 & -89 \\
\hline 9 & 113.60 & 113.60 & 36.75 & 146 & -88 \\
\hline 10 & 7.10 & 7.10 & 63.50 & -229 & 12 \\
\hline 11 & 33.72 & 33.72 & 63.50 & -110 & 6 \\
\hline 12 & 60.35 & 60.35 & 63.50 & -11 & 1 \\
\hline 13 & 86.97 & 86.97 & 63.50 & 74 & -4 \\
\hline 14 & 113.60 & 113.60 & 63.50 & 150 & -8 \\
\hline 15 & 7.10 & 7.10 & 90.25 & -224 & 121 \\
\hline 16 & 33.72 & 33.72 & 90.25 & -105 & 105 \\
\hline 17 & 60.35 & 60.35 & 90.25 & -6 & 92 \\
\hline 18 & 86.97 & 86.97 & 90.25 & 79 & 81 \\
\hline 19 & 113.60 & 113.60 & 90.25 & 155 & 72 \\
\hline 20 & 7.10 & 7.10 & 117.00 & -220 & 232 \\
\hline 21 & 33.72 & 33.72 & 117.00 & $-10 t$ & 206 \\
\hline 22 & 60.35 & 60.35 & 117.00 & -1 & 185 \\
\hline 23 & 86.97 & 86.97 & 117.00 & 84 & 168 \\
\hline 24 & 113.60 & 113.60 & 117.00 & 160 & 154 \\
\hline
\end{tabular}

For this test of our inversion procedure, we employ only one plane containing reference points instead of three as mentioned in the introduction. We did this because the diagonal plane can be used to accurately map the chamber volume without the use of the front and back plane for the theoretical model.

The absolute and pixel coordinates for our theoretical model are listed in Table 1. Using these values, the leastsquares best-fit values of $A_{0} \ldots A_{M-1}$ and $B_{0} \ldots B_{M-1}$ were determined for $M=9,11,17,21$, and 25 . The pixel positions of Table 1 were then input into Eqs. (22) along with these optimal values of $A_{0} \ldots B_{M-1}$ approximate values of $x$ and $z$ were determined. These were then compared with the original reference point coordinates $(x, z)$ and the average error per point was determined. Last, the average error per point was converted into an equivalent number of pixels using the fact that the entire chamber width of $127 \mathrm{~mm}$ is imaged onto 512 pixels in the CCD camera.

The resulting average errors per point in the inversion process expressed in terms of equivalent number of pixels is given in Table 2 for $M=9,11,17,21$, and 25 terms of the inversion polynomial. Not surprisingly, the more terms in Eqs. (22) that are kept, the more accurate is our approximate inversion process. But, as a practical matter, models 3,4 , and 5 with 17,21 , and 25 terms achieve a calibration error less than the pixel quantization error. Weng et al. take a complementary point of view. They use exact equations 
Table 2 Theoretical results (right camera view) with pixel quantization error $=0.5$ pixels.

\begin{tabular}{ccc}
\hline \hline Model & Number of Terms & $\begin{array}{c}\text { Average Theoretical } \\
\text { Error (in pixels) }\end{array}$ \\
\hline 1 & 9 & 1.30 \\
2 & 11 & 0.72 \\
3 & 17 & 0.25 \\
4 & 21 & 0.16 \\
5 & 25 & 0.15 \\
\hline
\end{tabular}

that give $x_{R}$ and $z_{R}$ as a function of $x, y$, and $z$. Their exact equations contain many lens distortion parameters. When they want to find $x$ and $z$ given the values of $x_{R}, z_{R}$, and $y$, they do not use a polynomial approximation to the exact nonlinear equations. They solve the exact nonlinear equations iteratively. Thus, for synthetic and simulated data, the theoretical error of their method is zero to within the convergence criterion of their iteration procedure. For real data and real lenses, their theoretical error describes how successfully or unsuccessfully their exact formulas model the operation of real lenses.

\section{Experimental Validation (2-D)}

The hardware used to provide the experimental results includes an IBM ${ }^{\mathrm{TM}}$ compatible $90-\mathrm{MHz}$ Pentium computer interfaced with a Recognition Technology Incorporated ${ }^{\mathrm{TM}}$ (RTI) image analysis subsystem and two SonyTM 3-chip CCD video cameras (Fig. 2). The images examined were $512 \times 512$ pixels with $512 \times 480$ pixels viewable and accessible using the RTI system.

We chose to conduct a testbed experiment on the placement and number of points to use in a typical experiment by constructing four separate calibration tests on volumes

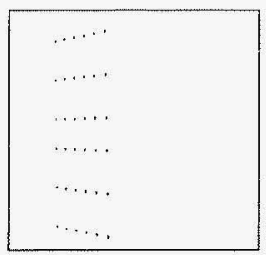

Front Plane

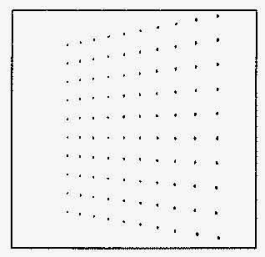

Center Plane

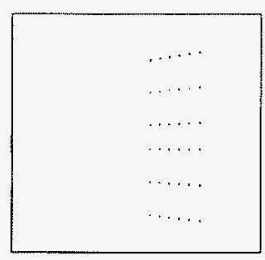

Rear Plane
Fig. 3 CCD image of the front, center, and rear calibration planes.

of $3,4,5$, and 6 in. This shows how well we can calibrate various sized volumes and how consistent a result we can obtain. For each experiment, we use a grid spacing of $1 / 4$ in. vertically and horizontally. This spacing was chosen after a series of tests on the 3-in. experimental volume to determine an optimal value. We used three diagonal calibration planes (Fig. 3) in each experiment and this yielded a total of 112 points for the 3-in. experiment, 119 points for the 4-in. experiment, 180 points for the 5 -in. experiment, and 275 points for the 6 -in. experiment.

The results of the calibrations are listed in Tables 3 through 6 . The results show that the calibration routine error is accurate and consistent by the average error varying from 1.16 to 2.49 pixels or 0.62 to $0.77 \%$ of full field for the left and right errors. Considering the fact that each particle is assumed to be between 3 and 5 pixels in diameter, we have achieved an average error less than one particle, which is a limiting factor in a typical experiment.

\section{Experimental Validation (3-D)}

We must combine the left and right calibrations to produce a 3-D camera calibration. This can be done by using Eqs. (22). In Eqs. (22), which represents the right camera cali-

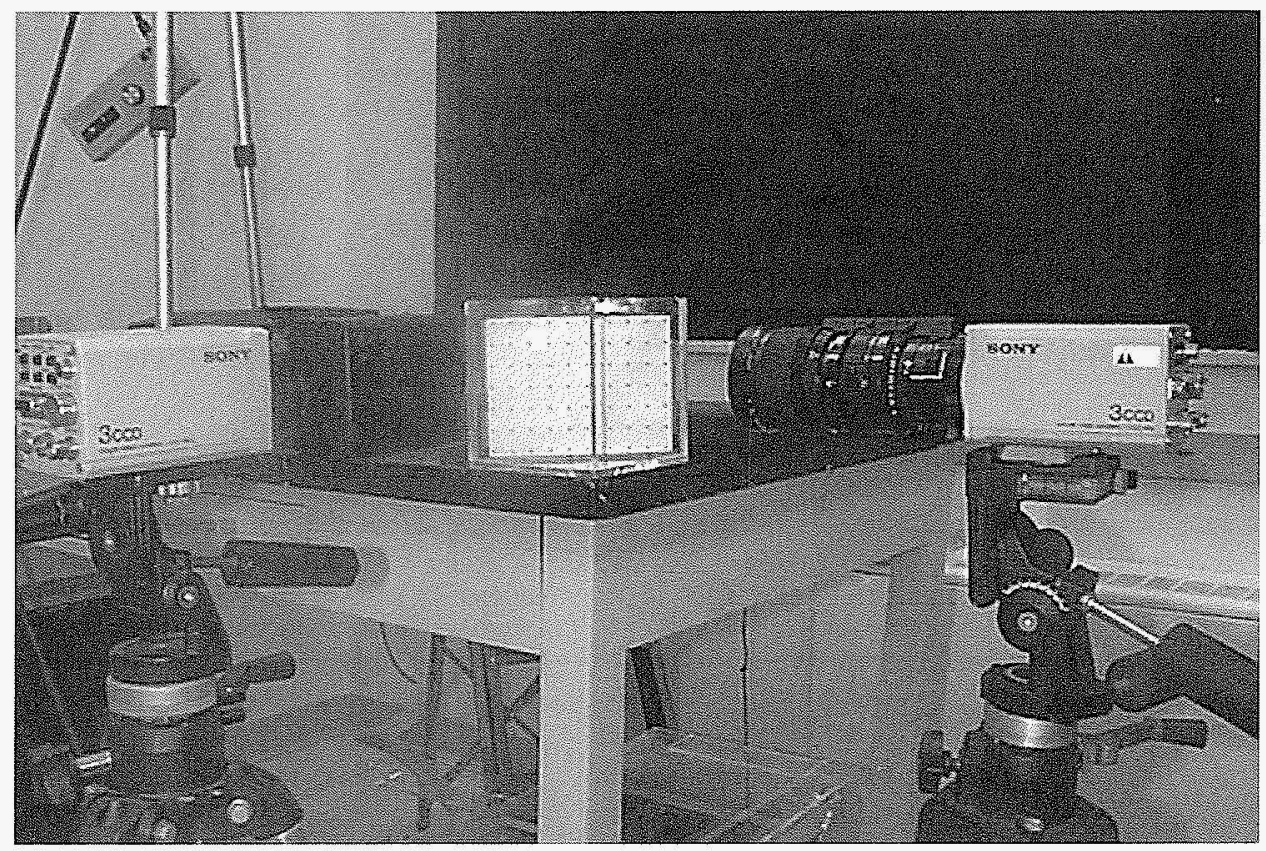

Fig. 2 Experimental camera calibration setup. 
Table 3 2-D calibration results for 3-in. volume with pixel quantization $=0.380 \mathrm{~mm} /$ pixel.

\begin{tabular}{ccc}
\hline \hline $\begin{array}{c}\text { Number } \\
\text { of Terms }\end{array}$ & $\begin{array}{c}\text { Left Camera } \\
\text { Average Error (2-D) } \\
\text { (in pixels) }\end{array}$ & $\begin{array}{c}\text { Right Camera } \\
\text { Average Error (2-D) } \\
\text { (in pixels) }\end{array}$ \\
\hline 9 & 2.01 & 1.71 \\
11 & 2.00 & 1.74 \\
17 & 1.89 & 1.73 \\
21 & 1.75 & 1.69 \\
25 & 1.71 & 1.66 \\
\hline \hline
\end{tabular}

bration parameters, the values for $x_{R}$ and $z_{R}$ are known and the value for $y$ is, in fact, unknown (contrary to the situation of the 2-D calibration in Secs. 3 to 5). We use the variable $t$ to represent depth (unknown $y$ variable) in the right camera and the variable $\tau$ to represent depth (unknown $x$ variable) in the left camera. Equations (22) for the right and left cameras can then be written compactly as

$x=A+B t$,

$y=t$,

$z=C+D t$

and

$x=\tau$,

$y=\alpha+\beta \tau$,

$z=\gamma+\delta \tau$,

where the variables $A, C, \alpha$, and $\gamma$ are used to group the terms in Eqs. (22) that do not involve $t$ or $\tau$. The variables $B, D, \beta$, and $\delta$ are used to group the terms in Eqs. (22) that involve $t$ and $\tau$.

We need to solve for $t$ and $\tau$, which represent the depth terms in the left and right views ( $Y Z$ and $X Z$ views), respectively. We interpret Eqs. (27) as a function of the parameter $t$, as seen by the right camera, and Eqs. (28) as a function of the parameter $\tau$, as seen by the left camera, as two rays propagating through the experimental chamber and representing the same particle. Ideally, these two rays

Table 4 2-D calibration results for 4-in. volume for pixel quantization $=0.362 \mathrm{~mm} /$ pixel.

\begin{tabular}{ccc}
\hline \hline $\begin{array}{c}\text { Number } \\
\text { of Terms }\end{array}$ & $\begin{array}{c}\text { Left Camera } \\
\text { Average Error (2-D) } \\
\text { (in pixels) }\end{array}$ & $\begin{array}{c}\text { Right Camera } \\
\text { Average Error (2-D) } \\
\text { (in pixels) }\end{array}$ \\
\hline 9 & 1.86 & 1.51 \\
11 & 1.86 & 1.51 \\
17 & 1.62 & 1.27 \\
21 & 1.62 & 1.26 \\
25 & 1.62 & 1.26 \\
\hline
\end{tabular}

Table 5 2-D calibration results for 5 -in. volume for pixel quantization $=0.388 \mathrm{~mm} /$ pixel.

\begin{tabular}{ccc}
\hline \hline $\begin{array}{c}\text { Number } \\
\text { of Terms }\end{array}$ & $\begin{array}{c}\text { Left Camera } \\
\text { Average Error (2-D) } \\
\text { (in pixels) }\end{array}$ & $\begin{array}{c}\text { Right Camera } \\
\text { Average Error (2-D) } \\
\text { (in pixels) }\end{array}$ \\
\hline 9 & 1.34 & 1.20 \\
11 & 1.34 & 1.20 \\
17 & 1.32 & 1.20 \\
21 & 1.32 & 1.18 \\
25 & 1.29 & 1.16 \\
\hline \hline
\end{tabular}

intersect at the particle position, but, due to experimental error, the right view ray passes close to the left view ray rather than intersecting it. For this case, we associate a pair of rays with each other in the following way. For a given ray in the right view, we find the left view ray that is closest to it and label this pair as a possible match describing the same particle. Then, for a given ray in the left view, we find the right view ray that is closest to it. If this pair is identical to the pair identified on the first pass through the data, we consider the match as definite. We take the midpoint of the common normal to the rays as the position of the particle, as shown in Fig. 4.

Once the two rays corresponding to a given particle are identified, the position of the particle is determined as follows. The directions along the right ray and the left ray are

$\mathbf{V}_{R}=B \hat{u}_{x}+1 \hat{u}_{y}+D \hat{u}_{z}$

$\mathbf{V}_{L}=1 \hat{u}_{x}+\beta \hat{u}_{y}+\delta \hat{u}_{z}$.

The plane containing these two rays has the normal $\mathbf{N}$ given by

$\mathbf{V}_{R} \times \mathbf{V}_{L}=\mathbf{N}$

or

$\mathbf{N}=\hat{u}_{x}(\delta-\beta D)+\hat{u}_{y}(B \delta-D)+\hat{u}_{z}(B \beta-1)$.

The line in the $\mathbf{N}$ direction passing through the point $t$ on the right ray is

$\frac{x-(A+B t)}{a}=\frac{y-t}{b}=\frac{z-(C+D t)}{c}$,

Table 6 2-D calibration results for 6 -in. volume for pixel quantization $=0.430 \mathrm{~mm} /$ pixel.

\begin{tabular}{ccc}
\hline \hline $\begin{array}{c}\text { Number } \\
\text { of Terms }\end{array}$ & $\begin{array}{c}\text { Left Camera } \\
\text { Average Error (2-D) } \\
\text { (in pixels) }\end{array}$ & $\begin{array}{c}\text { Right Camera } \\
\text { Average Error (2-D) } \\
\text { (in pixels) }\end{array}$ \\
\hline 9 & 2.10 & 2.49 \\
11 & 2.05 & 2.49 \\
17 & 1.98 & 2.36 \\
21 & 1.93 & 2.36 \\
25 & 1.91 & 2.36 \\
\hline
\end{tabular}


Bethea et al.: Three-dimensional camera calibration technique ...

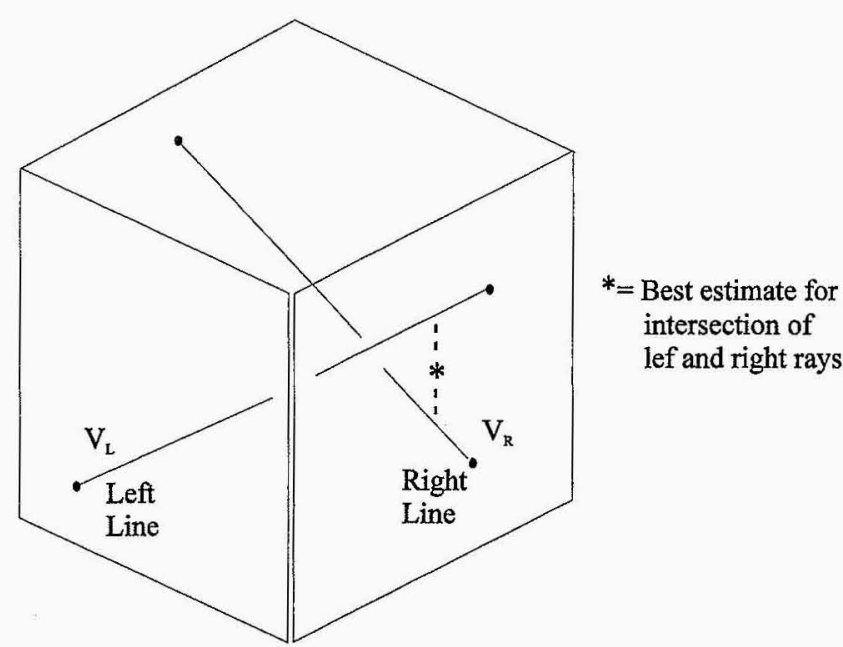

Fig. 4 Intersection of two rays.

where

$a=\delta-\beta D$,

$b=B \delta-D$

$c=B \beta-1$

This line passes through the point $\tau$ on the left ray when

$\frac{\tau-(A+B t)}{a}=\frac{(\alpha+\beta \tau)-t}{b}=\frac{(\gamma+\delta \tau)-(C+D t)}{c}$

This represents two linear equations in the two unknowns $t$ and $\tau$ whose solution is

$$
\begin{aligned}
& t=\frac{a \alpha+a \beta \tau-b \tau+b A}{a-b B} \\
& \tau=\frac{a \gamma-b B \gamma-a C+b B C-a D \alpha-b A D+c A+c B \alpha}{c-c B \beta-a \delta+b B \delta+a D \beta-b D}
\end{aligned}
$$

Substitution of these values of $t$ and $\tau$ into Eqs. (27) and (28) gives the two estimates of the absolute coordinates of the seed particle. Averaging these two results corresponds to the midpoint of the line $t \tau$ in Fig. 4. The results of

Table 7 3-D calibration results for 3 -in. volume for pixel quantization $=0.380 \mathrm{~mm} /$ pixel.

\begin{tabular}{cc}
\hline \hline $\begin{array}{c}\text { Number } \\
\text { of Terms }\end{array}$ & $\begin{array}{c}\text { Left and Right Camera } \\
\text { Average Error (3-D) } \\
\text { (in pixels) }\end{array}$ \\
\hline 9 & 2.13 \\
11 & 2.12 \\
17 & 2.08 \\
21 & 1.92 \\
25 & 1.87 \\
\hline \hline
\end{tabular}

Table 8 3-D calibration results for 4 -in. volume for pixel quantization $=0.362 \mathrm{~mm} /$ pixel.

\begin{tabular}{cc}
\hline \hline $\begin{array}{c}\text { Number } \\
\text { of Terms }\end{array}$ & $\begin{array}{c}\text { Left and Right Camera } \\
\text { Average Error (3-D) } \\
\text { (in pixels) }\end{array}$ \\
\hline 9 & 2.05 \\
11 & 2.05 \\
17 & 1.82 \\
21 & 1.80 \\
25 & 1.79 \\
\hline \hline
\end{tabular}

combining the two 2-D calibrations (left and right) to produce one 3-D camera calibration are given in Tables 7 to 10 . The results show that the $3-\mathrm{D}$ calibration results yield between 1.68 and 3.13 pixels or 0.48 to $1.01 \%$ of the full field 3-D error. Since each particle is assumed to be between 3 and 5 pixels, we have achieved a 3-D error of less than one particle (worst case) using our experimental chambers.

As stated in the introduction, we compare our calibration error to the NSCE error of Weng et al. and get an NSCE error of 0.6785 , which corresponds to a triangulation error that is lower, on average, than the digitization noise of a pixel at this depth in the field of view. Thus, this approach yields an accurate and reliable 3-D camera calibration routine by combining two 2-D calibrations positioned 90 deg apart."

\section{Summary and Conclusions}

A 3-D camera calibration technique has been developed by combining two 2-D camera calibrations for cameras positioned 90 deg apart (orthogonal stereo viewing). The left camera view ( $Y Z$ view) and the right camera view ( $X Z$ view) are calibrated separately and then combined to produce an $X Y Z$ (3-D) calibration routine. The technique is based on using three parallel calibration planes placed inside a volume so that both cameras can view the calibration points simultaneously. We chose the positions of a number of calibration points in a volume (absolute coordinates $x$, $y$, and $z$ ) and used a physical model to determine the exact pixel locations of the calibration points. We then input the absolute coordinates and pixel locations into a least-squares fitting algorithm to obtain the experimental camera parameters. When analyzing our theoretical model, a camera calibration accuracy of less than 1.30 pixels was achieved.

Table 9 3-D calibration results for 5-in. volume for pixel quantization $=0.388 \mathrm{~mm} /$ pixel.

\begin{tabular}{cc}
\hline $\begin{array}{c}\text { Number } \\
\text { of Terms }\end{array}$ & $\begin{array}{c}\text { Left and Right Camera } \\
\text { Average Error (3-D) } \\
\text { (in pixels) }\end{array}$ \\
\hline 9 & 1.72 \\
11 & 1.71 \\
17 & 1.70 \\
21 & 1.70 \\
25 & 1.68 \\
\hline \hline
\end{tabular}


Table $103-D$ calibration results for 6 -in. volume for pixel quantization $=0.430 \mathrm{~mm} /$ pixel

\begin{tabular}{cc}
\hline $\begin{array}{c}\text { Number } \\
\text { of Terms }\end{array}$ & $\begin{array}{c}\text { Left and Right Camera } \\
\text { Average Error (3-D) } \\
\text { (in pixels) }\end{array}$ \\
\hline 9 & 3.28 \\
11 & 3.27 \\
17 & 3.20 \\
21 & 3.15 \\
25 & 3.13 \\
\hline
\end{tabular}

When analyzing our 2-D experimental model, a camera calibration accuracy between 1.16 and 2.49 pixels or 0.62 to $0.77 \%$ of full field for the left and right errors was achieved. When combining the left and right views to produce one 3-D calibration, an accuracy yield between 1.68 and 3.13 pixels or 0.48 to $1.01 \%$ of full field was achieved. All of the camera calculations were done on a Pentiumbased computer and a typical calibration from start to finish usually takes less than 10 min.

In addition to completing a testbed experiment in Secs. 7 and 8 , we have also conducted calibration tests on volumes of $0.6 \mathrm{in}^{3}\left(1.52 \mathrm{~mm}^{3}\right), \quad 1 \mathrm{in}^{3}\left(2.54 \mathrm{~mm}^{3}\right)$, and $3 \mathrm{ft}^{3}$ $\left(91.44 \mathrm{~mm}^{3}\right)$ for industrial applications and achieved similar calibration results. The results show that the camera calibration routine is mathematically sound and experimentally verified and can be easily implemented into any existing 3-D experiment to produce accurate quantitative information.

\section{Acknowledgment}

$n$,

The 3-D camera calibration discussed in this paper is currently used in computer vision experiments in the Stereo Imaging Velocimetry Laboratory at the National Aeronautics and Space Administration Lewis Research Center.

\section{References}

1. R. Y. Tsai, "A versatile camera calibration technique for high accuracy 3D machine vision metrology using off-the-shelf TV camera and lenses "IEEE J. Robot. Automat. RA-3 323-344 (1987).

2. R. Y. Tsai, "Techniques for calibration of the scale factor and image center for high accuracy 3D machine vision metrology," IEEE Proc. Int. Conf. on Robotics and Automation, (1987).

3. J. Weng, P. Cohen, and M. Herniou, "Camera calibration with distortion models and accuracy evaluation," IEEE Trans. Pattern Anal. Mach. Intell. 14, 965-980 (1992).

4. H. A. Martins, J. R. Dirk, and R. B. Kelly, "Camera models based on data from two calibration planes," Comput. Graph. Image Process. 17. $173-180(1981)$.

5. B. Kamgar-Parsi and R. D. Eastman, "Calibration of a stereo system with small relative angles," Comput. Vis. Graph. Image Process. 51, $1-19(1990)$.

6. A. A. Adamczyk and L. Rimai, "Reconstruction of a 3-dimensional flow field from orthogonal views of seed track video images," Exper. Fluids 6, 380-386 (1988)

7. R. G. Racca and J. M. Dewey, "A method for automatic particle tracking in a three-dimensional fow field," Exper. Fluids 6, 25-32 (1988).

8. T. Kobayashi, T. Saga, and K. Sekimoto, "Velocity measurement of three-dimensional flow around rotating parallel disks by digital image processing." in Proc. ASME Winter Ann. Meet.. San Francisco (1989).

9. K. Nishino, N. Kasagi, and M. Hirata, "Three-dimensional particle tracking velocimetry based on automated digital image processing." Trans. ASME, 111, 384-39] (1989).

10. B. B. Miller, M. B. Meyer, and M. D. Bethea, "Stereo imaging velocimetry for microgravity applications." presented at the Int. Symp. on Space Optics, 1994, Garmisch-Partenkirchen, FRG, EOS and SPIE.

11. M. B. Meyer, M. D. Bethea, Y. G. Guezennec, and W. C. Choi, "Stereo imaging velocimetry: a new option for microgravity experiments," in Proc. ASME Winter Ann. Meet. AMD-Vol. 174/FED-Vol. 175, Fluid Mechanics Phenomena in Microgravity, pp. 173-182, New Orleans, (1993).

12. M. B. Meyer and M. D. Bethea, "A full field, 3-D velocimeter for NASA's microgravity science program," presented at AIAA 30th Aerospace Sciences Meet. and Exhibit, 1992, AIAA-92-0784.

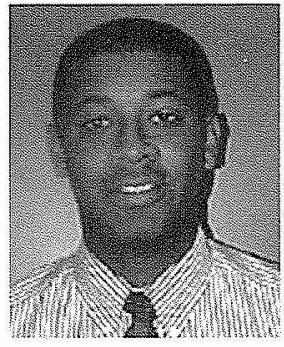

Mark D. Bethea received a BS in mathematics/computer science in 1987, a $B S$ in mathematics education and a BS in pure mathematics, both in 1988, from Delaware State University in Dover, Delaware. He received an MS in applied mathematics in 1989 from Cleveland State University in Cleveland, Ohio. He received a $\mathrm{PhD}$ in computer engineering in 1996 from Case Western Reserve University in Cleveland, Ohio. He is currently a project scientist at the NASA Lewis Research Center, Cleveland, Ohio. His research interests are stereo imaging velocimetry, fluid physics, digital machine vision, and artifical neural networks.

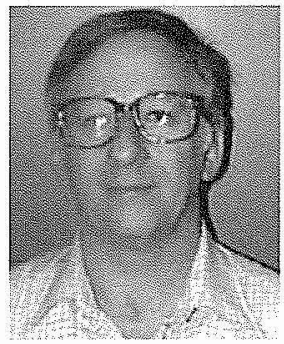

James A. Lock received his $B S$ and $P h D$ degrees in physics from Case Western Reserve University in 1970 and 1974, respectively. He has been a faculty member in the Physics Department at Cleveland State University in Cleveland, Ohio, since 1978. His main research interest is light scattering. He is a topical editor of the Journal of Applied Optics.

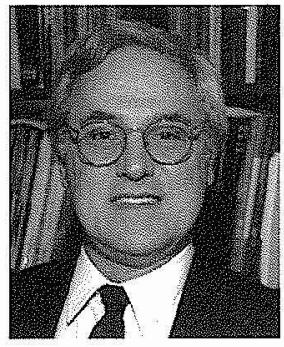

Frank Merat is an associate professor of electrical engineering and applied physics at Case Western Reserve University, Cleveland, Ohio. Dr. Merat received his professional education in electrical engineering, a BS in 1972, MS in 1975, and $\mathrm{PhD}$ in 1978, all from Case Western Reserve University. Dr. Merat's current research interests include MEMS for optical appliations, neural network signal processing for MEMS sensor arrays, computer vision for flexible manufacturing, biologically inspired signal and image processing, and optical sensors. His previous work has included electronic holography, 3-D holographic displays, NMM lasers and imaging, and concurrent design. Dr. Merat is a member of IEEE, SPIE, the Association for Computing Machinery, and the Machine Vision Association of the Society of Manufacturing Engineers. $\mathrm{He}$ is a past chairman of the Cleveland Section of IEEE, and is currently executive officer of the Department of Electrical Engineering at Case Western Reserve University.

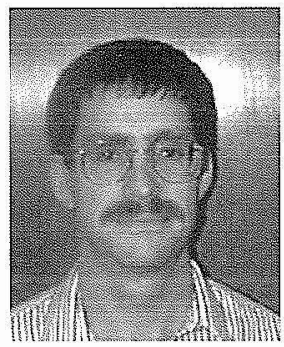

Paul D. Crouser received a BS in chemistry from Oberlin College and an MS in computer science from Cleveland State University. $\mathrm{He}$ is currently a $\mathrm{PhD}$ candidate in the Department of Computer Engineering and Science at Case Western Reserve University, Cleveland, Ohio. He is the lead project engineer for the Stereo Imaging Velocimetry project at the NASA Lewis Research Center, Cleveland, Ohio. $\mathrm{He}$ is also a project engineer at AYT Corporation, Cleveland, Ohio, developing a neutral net controller to control acoustic buzz in jet engines. His research interests are in developing state of the art image recognition systems. 